\title{
Charged lepton flavour violating processes
}

\author{
Adrian Signer ${ }^{1,2 \star}$ \\ 1 Paul Scherrer Institut, CH-5232 Villigen PSI, Switzerland \\ 2 Physik-Institut, Universität Zürich, CH-8057 Zürich, Switzerland \\ $\star$ adrian.signer@psi.ch \\ Proceedings for the 15th International Workshop on Tau Lepton Physics, \\ Amsterdam, The Netherlands, 24-28 September 2018 \\ doi:10.21468/SciPostPhysProc. 1

\begin{abstract}
Charged lepton flavour violating processes are naturally present in many extensions of the Standard Model. After a brief overview on the experimental situation, an effectivefield-theory framework is described that allows to interpret and compare the various experiments in a consistent way. The usefulness of this approach is then illustrated in the context of a specific model with a doubly charged scalar.
\end{abstract}

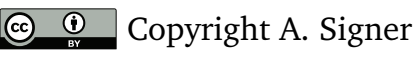

This work is licensed under the Creative Commons

Attribution 4.0 International License.

Published by the SciPost Foundation.
Received 10-12-2018

Accepted 17-01-2019

Published 19-02-2019

doi:10.21468/SciPostPhysProc.1.014

\section{Introduction}

In the Standard Model (SM) without right-handed neutrinos, lepton flavour is conserved. This is an accidental symmetry simply due to the fact that it is not possible to write down a renormalizable and gauge-invariant operator that violates (charged) lepton flavour. In the quark sector the situation is different. Since all quarks have non-vanishing (and different) masses, the distinction between mass eigenstates and interaction eigenstates leads to "quark flavour violation".

If we take into account neutrino masses, lepton flavour is also violated. It is true that taken alone, this violation is very weak in the sense that it leads to branching ratios for charged lepton flavour violating (cLFV) decays that are unobservable in any conceivable experiment. However, the crucial message is that as for quark flavour, there is nothing sacred about lepton flavour conservation.

As a consequence, beyond the Standard Model (BSM) physics will lead to cLFV unless special care is taken to prevent it. The fact that we have not seen it yet despite substantial experimental effort has two possible explanations: either BSM has some mechanism to prevent CLFV, or the new physics (NP) scale related to CLFV is so high that the corresponding interactions are extremely suppressed at the low-energy scale of the experiments. The first option is actually a step back from the SM. While the absence of observable cLFV has a natural explanation within the SM, going beyond invalidates this and leads to a problem that was not present in the SM. Creating new problems is not what we usually expect from BSM physics. 
The second option is much more natural, albeit somewhat sobering. Taken at face value, it leads to the expectation of NP at an extremely high scale (at or above $10^{10} \mathrm{GeV}$ ). If this was the case, neutrino masses would be the NP we observe and cLFV is the next best chance, but direct searches at the high-energy frontier are bound to fail. The only consolation in this scenario is that there is no reason for there to be only a single scale of NP. Indeed, currently the $B$-anomalies for example offer a fragile glimmer of hope that other NP is present at much smaller scales (see e.g. [1]).

In either case, the investigation of cLFV processes offers the possibility to test the SM to very high scales or actually find BSM. Since this will be indirect evidence, it is crucial to consider as many observables as possible. Indirect evidence from a single observable will at most tell us "there is something", but never give enough information to conclude what it is. A comprehensive study of cLFV however will be able to seriously constrain the infinitely many options of BSM and, in the best of worlds, point us to the option nature has chosen.

\section{Observables}

There is a plethora of processes where we can look for cLFV effects. The most stringent limits have been obtained for the golden muon decay channels, namely $\operatorname{Br}(\mu \rightarrow e \gamma)<4.2 \times 10^{-13}$ [2], $\operatorname{Br}(\mu \rightarrow 3 e)<1.0 \times 10^{-12}$ [3] and muon conversion in the field of a nucleus. As an example of the latter, for muon conversion in a gold atom the limit is $\mathrm{Br}_{\mu \rightarrow e}^{\mathrm{Au}}<7 \times 10^{-13}$ [4], where the decay rate is normalized by the capture rate. As I will argue below, the limits on these observables are highly complementary and improving the limits on all of these observables will provide important information. Luckily, the near future will see a lot of experimental progress. MEG II will improve the limit on $\mu \rightarrow \mathrm{er}$ by an order of magnitude [5], while Mu3e will increase the significance on $\mu \rightarrow 3 e$ by several orders of magnitude [6]. For muon conversion, COMET [7] and Mu2e [8] will also improve the sensitivity by about four orders of magnitude, using aluminium.

Given that the SM rate for these processes are tiny it is often said that these searches are background free. However, there is of course background from 'boring' SM processes. Apart from the reducible (accidental) background, which can be tackled by improving the resolution of the detectors, there is the irreducible background. For $\mu \rightarrow e \gamma$ and $\mu \rightarrow 3 e$ this is due to SM processes $\mu \rightarrow e \gamma \nu \bar{v}$ and $\mu \rightarrow 3 e v \bar{v}$ in the region where the neutrinos have very little energy. These processes have by now all been calculated at NLO in the electromagnetic coupling, providing reliable predictions to estimate the number of irreducible background events [9-14]. For muon conversion, an irreducible background appears through the decay of the muon in orbit. Since this decay happens in the field of the nucleus, the energy distribution of the emitted electron has a tail up to the muon mass (minus binding energy). Again, there exist reliable theoretical predictions for this decay [15]. For all cases the bottom line is that the irreducible background is not preventing measurements with the proposed sensitivity.

Apart from muon decays, also tau decays offer a very rich field to study cLFV. Typically, the experimental constraints are weaker by several orders of magnitude. For example, the limits on the various branching ratios for $\tau \rightarrow 3 \ell$ with $\ell \in\{e, \mu\}$ are all about $\operatorname{Br}(\tau \rightarrow 3 \ell) \lesssim 2 \times 10^{-8}$ [16] and the limits for $\tau \rightarrow \ell \gamma$ are roughly $\operatorname{Br}(\tau \rightarrow \ell \gamma) \lesssim 4 \times 10^{-8}[17,18]$. However, in the case of the $\tau$ there are numerous further LFV decays possible and many have strong experimental constraints. To mention but a few, we have e.g. $\operatorname{Br}\left(\tau \rightarrow \ell \pi^{0}\right) \lesssim 1 \times 10^{-7}[19,20]$, $\operatorname{Br}\left(\tau \rightarrow \ell K_{S}^{0}\right) \lesssim 3 \times 10^{-8}[21,22]$ and $\operatorname{Br}\left(\tau \rightarrow \ell K^{+} K^{-}\right) \lesssim 4 \times 10^{-8}$ [23]. Once Belle II is in operation, many of these branching ratios will be scrutinized even more stringently. Should there ever be evidence for NP in one of these channels the presence of similar observables will be very beneficial to assess the reliability of the evidence as well as constraining possible BSM 
explanations.

\section{Looking for BSM}

The main motivation to investigate cLFV is to constrain the SM and look for BSM. Since cLFV will only give indirect information it is necessary to compare the information on the branching ratios to a hypothesis. Ultimately, of course we want to find the ultraviolet (UV) complete theory that describes BSM physics and compare its predictions to cLFV data. Unfortunately, choosing the appropriate UV complete theory requires divine inspiration, resulting in very specific case by case studies in this approach.

In order to be more general, it is possible to consider so-called simplified models. Without worrying about UV completion new particles (typically scalars or vectors) are introduced. The degrees of freedom then are the mass of the new particle and its couplings to SM particles and constraints on these parameters are obtained through cLFV data.

Making the approach even more general we can work with effective theories. In this case no new dynamical degrees of freedom are introduced at all and the effects of BSM physics are encoded in Wilson coefficients of higher-dimensional operators. The latter are constructed from SM fields, ensuring gauge and Lorentz invariance.

In practice, the most promising approach is probably bottom up, i.e. starting from an effective theory. Once a pattern of deviations in Wilson coefficients is established, a simplified model that produces these coefficients can be looked for. In case such a simplified model can be found, its embedding in a UV complete theory would be the final step. In fact, currently precisely such a programme has been started in the context of $B$ anomalies.

In the reminder we will focus on the effective field theory (EFT) approach and study its interplay with an explicit BSM model. For the latter we will consider as an example the simple case of a doubly-charged scalar. The standard expectation of BSM physics is that it is related to new heavy degrees of freedom at a scale $\Lambda$, considerably above the electroweak (EW) scale $\Lambda \gg M_{e w}$. On the other hand we are using experiments at much smaller scales $\left\{m_{\mu}, m_{\tau}\right\} \ll M_{e w} \ll \Lambda$. Thus, it is very natural to use an effective theory where the (unknown) heavy degrees of freedom have been integrated out. After all, EFT are precisely made for situations with two (or more) widely different scales. In fact, we have to use two different EFT. Integrating out NP at the scale $\Lambda$ will lead to a tower of higher-dimensional operators which respect the gauge symmetries of the $S M, S U_{c}(3) \times S U_{L}(2) \times U_{Y}(1)$. This EFT is called SMEFT. However, for muon and tau decays, also the degrees of freedom with EW scale can (and should) be integrated out. Below the EW scale, the symmetry of the theory is $S U_{c}(3) \times U_{e m}(1)$, and to be completely general, all (Lorentz invariant) operators respecting this symmetry should be included. Of course, in $B$ physics, such an effective theory has been used for decades. In the context of cLFV, we will refer to it as LEFT (low energy EFT).

\section{Effective theory above the electroweak scale}

The Lagrangian of SMEFT has a single operator of dimension five [24], related to neutrino masses. Including also dimension 6 operators [25] we write

$$
\mathcal{L}_{S M E F T}=\mathcal{L}_{S M}+\frac{1}{\Lambda} C^{(5)} Q^{(5)}+\frac{1}{\Lambda^{2}} \sum C_{i}^{(6)} Q_{i}^{(6)}+\ldots
$$

The Wilson coefficients at the high scale, $C_{i}^{(6)}(\Lambda)$, directly encode the information of the BSM theory and can be considered as a parameterization of our ignorance. However, it cannot be 
stressed enough that the Lagrangian of (1) describes a quantum field theory that allows to perform perturbative computations, in principle to any order in the couplings. Thus, the couplings $C_{i}^{(6)}(\mu)$ do depend on the scale and this dependence is governed by renormalization group equations (RGE) resumming large logarithms $\log (\Lambda / \mu)$. The various operators mix under the RGE. Hence a particular coupling $C_{i}^{(6)}\left(M_{e w}\right)$ does in general depend on many $C_{j}^{(6)}(\Lambda)$.

A first example of the impact of RGE in the context of cLFV processes has been considered in [26] where the branching ratio for $\mu \rightarrow e \gamma$ has been evaluated at leading order in $1 / \Lambda^{2}$, but taking into account one-loop RGE. Doing this, $\operatorname{Br}(\mu \rightarrow e \gamma)$ does not only depend on the coefficient $C_{e \gamma}^{(6)}$ of the dipole operator (expressed in terms of left-handed doublets $l$, righthanded singlets $e$ and the Higgs doublet $\Phi$ )

$$
\left(Q_{e \gamma}^{(6)}\right)_{i j}=\left(\bar{l}_{i} \sigma^{\mu v} e_{j}\right) \Phi F_{\mu \nu},
$$

with family indices $i, j \in\{1,2\}$, but also on some four-fermion operators. Limits are always obtained for $C_{i}^{(6)} / \Lambda^{2}$. Thus, any statement about probing energy scales of a certain order relies on an assumption about the size of the couplings $C_{i}^{(6)}$. If, for some weird reason, we expect them to be $C_{i}^{(6)} \sim 1$, the energy range probed through the dipole coupling is $\Lambda \gtrsim 10^{3} \mathrm{TeV}$ and the best constrained four-fermion interaction is only slightly weaker. In particular, it should be noted that limits on some four-fermion interactions due to $\mu \rightarrow e \gamma$ can be extremely stringent. Thus, the continuously repeated statement that $\mu \rightarrow e \gamma$ does not constrain contact interactions has to be strongly refuted.

Of course, processes other than $\mu \rightarrow e \gamma$ can be considered as well and this approach allows to relate processes like $\ell_{i} \rightarrow \ell_{j} \gamma$ for example to $Z \rightarrow \ell_{i} \ell_{j}$. Another interesting option is to consider anomalous magnetic moments or electric dipole moments of leptons in such an approach [27]. In fact, the one-loop anomalous dimensions for all dimension 6 operators of SMEFT have been computed $[28,29]$. This allows for the evaluation of the leading logarithms $\log \left(\Lambda / M_{\text {ew }}\right)$ associated with effects of dimension 6 operators for all observables at the EW scale.

\section{Effective theory below the electroweak scale}

The formalism described in the previous section allows to relate the SMEFT Wilson coefficients at the high scale, $C_{i}^{(6)}(\Lambda)$, to observables at the EW scale, resumming $\log \left(\Lambda / M_{e w}\right)$. For processes at the LHC, this is precisely what is needed. However, the processes we are concerned with here take place at an even lower scale. Thus, the appropriate EFT is not SMEFT, but LEFT, an EFT that contains only light fermions, gluons and photons. Furthermore, there is no $S U_{L}(2)$ symmetry any longer. Thus the higher-dimensional operators of LEFT, $\mathcal{Q}_{i}$ are not expressed in terms of $S U_{L}(2)$ doublets $l$ any longer but in terms of left- and right-handed singlet fermion fields, $e_{L}$ and $e_{R}$. Looking at four-fermion interactions, this disentangles neutrino interactions from the corresponding charged lepton interactions. SMEFT four-fermion operators are split into LEFT four-fermion operators like e.g.

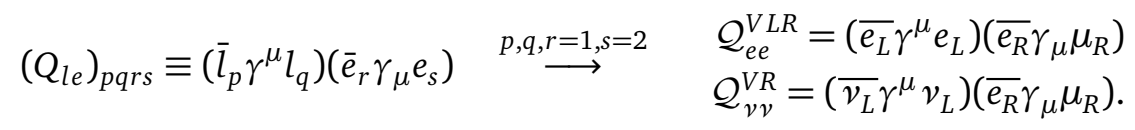

If we keep the assumption that NP enters at a scale $\Lambda \gg M_{e w}$ we obtain $\mathcal{L}_{L E F T}$ from $\mathcal{L}_{S M E F T}$ through matching at the EW scale [30], i.e. we can express $\mathcal{C}\left(M_{e w}\right)$ in terms of $C\left(M_{e w}\right)$. However, we can even consider the option that NP actually enters at a scale between the tau mass and the EW scale. In this way, the Wilson coefficients of LEFT such as $\mathcal{C}_{e e}^{V L R}$ and $\mathcal{C}_{v v}^{V R}$ 
associated with the operators on the r.h.s. of (3) are truly independent. The formal expression for the Lagrangian

$$
\mathcal{L}_{L E F T}=\mathcal{L}_{Q E D}+\mathcal{L}_{Q C D}+\frac{1}{M_{e w}} \sum_{j} \mathcal{C}_{j}^{(5)} \mathcal{Q}_{j}^{(5)}+\frac{1}{M_{e w}^{2}} \sum_{i} \mathcal{C}_{i}^{(6)} \mathcal{Q}_{i}^{(6)}+\ldots
$$

however, is very similar to (1).

We can now express the observables at the (very low) physical scale through Wilson coefficients $\mathcal{C}\left(\left\{m_{\mu}, m_{\tau}\right\}\right)$ evaluated at this scale. To make connection to NP, we then relate $\mathcal{C}\left(\left\{m_{\mu}, m_{\tau}\right\}\right)$ to $\mathcal{C}\left(M_{e w}\right)$ or any other scale below the EW scale. For the golden channels this programme has been carried out in [31], including all relevant one-loop anomalous dimensions as well as the two-loop contribution into the dipole operator. As in SMEFT, the operators in LEFT mix under RGE. Hence, if expressed in terms of Wilson coefficients at the EW scale, different observables contribute to the same operators. This should be seen as a complementarity much more than a competition. Assuming a particular experimental search was to see a deviation, other observables are required to get a handle on what the cause is, i.e. which operator(s) are responsible.

The Lagrangian $\mathcal{L}_{\text {LEFT }}$ contains operators with light quark fields. Below a scale of about $1-2 \mathrm{GeV}$ the notion of $u, d$ and $s$ quark fields does not make sense any longer. Hence $\mathcal{L}_{L E F T}$ should be matched onto yet another effective Lagrangian where these operators are replaced by low-energy operators involving hadrons as in chrial perturbation theory. The effect of tensor operators in this context was studied recently [32].

\section{Interplay of a BSM model and the EFT approach}

As an example of how an explicit BSM is matched onto an EFT and then bounds on the Wilson coefficients are interpreted in terms of the underlying model we consider a doubly charged scalar (DCS) $S$ with the Lagrangian

$$
\begin{aligned}
\mathcal{L}_{\mathrm{UV}} & =\mathcal{L}_{\mathrm{SM}}+\left(D_{\mu} S^{++}\right)^{\dagger}\left(D^{\mu} S^{++}\right)+\left(\lambda_{a b} \overline{\left(\ell_{R}\right)_{a}^{c}}\left(\ell_{R}\right)_{b} S^{++}+\text {h.c. }\right)+ \\
& +\lambda_{2}\left(H^{\dagger} H\right)\left(S^{--} S^{++}\right)+\lambda_{4}\left(S^{--} S^{++}\right)^{2}+[\ldots] .
\end{aligned}
$$

We will focus on the couplings of the DCS to leptons, given by the symmetric matrix $\lambda_{a b}$, where $a$ and $b$ are flavour indices. As an example, we set the mass of the DCS to $m_{S}=1 \mathrm{TeV}$. In this case, we can integrate out the DCS together with the EW scale and match $\mathcal{L}_{\mathrm{UV}}$ directly to $\mathcal{L}_{L E F T}$. At the EW scale, this leads to a non-vanshing Wilson coefficient for some purely leptonic four-fermion operators (at tree level) as well as the leptonic dipole operator (at one loop). Through the RGE, this results also in four-fermion operators involving quarks [33]. It is absolutely crucial to take this effect into account, as it has a dramatic effect on low-energy observables, in particular on $\mu$-e conversion.

Given the couplings $\lambda_{a b}$ we can now predict all low-energy observables through the EFT and interpret the corresponding limits as limits on $\lambda_{a b}$. For illistrative purposes we will consider two examples. In the first, it is assumed that all couplings, $\lambda_{a b}^{D}$, are of the same order, possibly with a small hierarchy between diagonal and off-diagonal elements. In the second, we assume the couplings, $\lambda_{a b}^{Y}$, are Yukawa inspired, i.e. larger for the third family. Concretely, we use

$$
\lambda_{a b}^{D}=\lambda\left(\begin{array}{ccc}
1 & v & v^{2} \\
v & 1 & v \\
v^{2} & v & 1
\end{array}\right) \quad \lambda_{a b}^{Y}=\lambda\left(\begin{array}{ccc}
v^{2 d} & v^{d+1} & v^{d} \\
v^{d+1} & v^{2} & v \\
v^{d} & v & 1
\end{array}\right)
$$



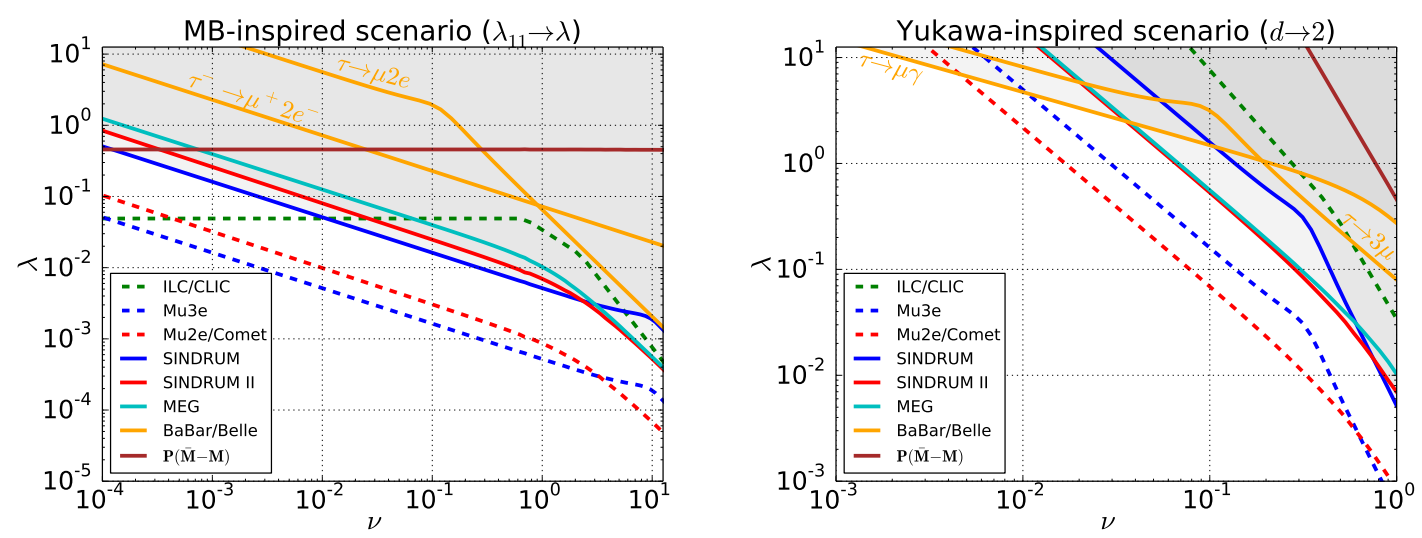

Figure 1: Limits on $\lambda$ and $v$ from various processes, using $\lambda_{a b}^{D}$ (left panel) and $\lambda_{a b}^{Y}$ (right panel) of(6) with $m_{S}=1$ TeV. Figures taken from [33].

with $d=2$ and typically $v<1$. As shown in the left panel of Figure 1, in the case of $\lambda_{a b}^{D}$ cLFV processes $\mu \rightarrow e$ are more restrictive than the corresponding processes involving $\tau$. This is simply due to the stronger experimental constraints. It has to be emphasised that $\mu$-e conversion is a powerful probe even though the DCS does not directly couple to quarks. The RGE generates this coupling at the low scale. This illustrates once more how important these effects are. Finally, input from a future linear collider can play an important role, in particular for small values of $v$, as indicated by the green dashed lines in the left panel of Figure 1.

If couplings to the third familiy are enhanced, processes involving $\tau$ become very competitive. This is illustrated by the orange lines in the right panel of Figure 1. In any case, it is clear that it is imperative to consider a wide variety of cLFV processes to optimize the analysing power of the experimental input.

\section{Conclusion}

Charged lepton flavour violating processes provide an opportunity to test the SM to extremely high energy scales of $\sim 100-1000 \mathrm{TeV}$. Despite having the reputation of being 'weird', in many extensions of the SM it is actually more natural to have them than to avoid them. In this talk (see [34] for a more general review) we have focused on an EFT treatment and given an example of how to link this with an explicit (simple) BSM model. Combining processes involving muons as well as taus offer the opportunity to severely constrain many BSM scenarios. With the huge experimental progress expected on many cLFV observables in the near future there is every reason to look forward to having another go at the fortress called Standard Model. Whether or not the recent $B$ anomalies survive, the flavour sector can be considered as a weak point of the SM and, hence, should be probed with as much gumption as possible.

\section{Acknowledgements}

The first five sections of this write-up are virtually identical to an earlier write-up of a talk [35]. This talk is based on the work presented in $[10,12,26,31,33]$ and I would like to thank my collaborators: first and foremost G. M. Pruna, as well as A. Crivellin, S. Davidson, M. Ghezzi, L. Panizzi and Y. Ulrich. 


\section{References}

[1] A. Crivellin et al., PSI/UZH Workshop: Impact of $B \rightarrow \mu^{+} \mu^{-}$on new physics searches (2018), arXiv:1803.10097.

[2] A. M. Baldini et al., Search for the lepton flavour violating decay $\mu^{+} \rightarrow \mathrm{e}^{+} \gamma$ with the full dataset of the MEG experiment, Eur. Phys. J. C 76, 434 (2016), doi:10.1140/epjc/s10052016-4271-x.

[3] U. Bellgardt et al., Search for the decay $\mu^{+} \rightarrow e^{+} e^{+} e^{-}$, Nucl. Phys. B 299, 1 (1988), doi:10.1016/0550-3213(88)90462-2.

[4] W. Bertl et al., A search for $\mu$ - e conversion in muonic gold, Eur. Phys. J. C 47, 337 (2006), doi:10.1140/epjc/s2006-02582-x.

[5] A. M. Baldini et al., The design of the MEG II experiment, Eur. Phys. J. C 78, 380 (2018), doi:10.1140/epjc/s10052-018-5845-6.

[6] N. Berger, The Muße Experiment, Nucl. Phys. B Proc. Suppl. 248-250, 35 (2014), doi:10.1016/j.nuclphysbps.2014.02.007.

[7] Y. G. Cui et al., Conceptual design report for experimental search for lepton flavor violating $\mu^{-}-e^{-}$conversion at sensitivity of $10^{-16}$ with a slow-extracted bunched proton beam (COMET), J-PARC P21 (2009).

[8] R. M. Carey et al., Proposal to search for $\mu^{-} N \rightarrow e^{-} N$ with a single event sensitivity below $10^{-16}$, FERMILAB-PROPOSAL-0973 (2008), doi:10.2172/952028.

[9] M. Fael, L. Mercolli and M. Passera, Radiative $\mu$ and $\tau$ leptonic decays at NLO, J. High Energ. Phys. 07, 153 (2015), doi:10.1007/JHEP07(2015)153.

[10] G. M. Pruna, A. Signer and Y. Ulrich, Fully differential NLO predictions for the rare muon decay, Phys. Lett. B 765, 280 (2017), doi:10.1016/j.physletb.2016.12.039.

[11] M. Fael and C. Greub, Next-to-leading order prediction for the decay $\mu \rightarrow e\left(e^{+} e^{-}\right) v \bar{v}, \mathrm{~J}$. High Energ. Phys. 01, 084 (2017), doi:10.1007/JHEP01(2017)084.

[12] G. M. Pruna, A. Signer and Y. Ulrich, Fully differential NLO predictions for the radiative decay of muons and taus, Phys. Lett. B 772, 452 (2017), doi:10.1016/j.physletb.2017.07.008.

[13] Y. Ulrich, Fully differential NLO predictions for rare and radiative lepton decays, Proc. Sci. 295, 124 (2018), doi:10.22323/1.295.0124.

[14] M. Fael, NLO prediction for the decays $\tau \rightarrow \ell \ell^{\prime} \ell^{\prime} v \bar{v}$ and $\mu \rightarrow e e e v \bar{v}$, SciPost Phys. Proc. 1, 009 (2018), doi:10.21468/SciPostPhysProc.1.009

[15] A. Czarnecki, X. Garcia i Tormo and W. J. Marciano, Muon decay in orbit: Spectrum of highenergy electrons, Phys. Rev. D 84, 013006 (2011), doi:10.1103/PhysRevD.84.013006.

[16] K. Hayasaka et al., Search for lepton flavor violating tau decays into three leptons with 719 million produced $\tau^{+} \tau^{-}$pairs, Phys Lett. B 687, 139 (2010), doi:10.1016/j.physletb.2010.03.037.

[17] K. Hayasaka et al., New search for $\tau \rightarrow \mu \gamma$ and $\tau \rightarrow$ er decays at Belle, Phys. Lett. B 666, 16 (2008), doi:10.1016/j.physletb.2008.06.056. 
[18] B. Aubert et al., Searches for lepton flavor violation in the decays $\tau^{ \pm} \rightarrow e^{ \pm} \gamma$ and $\tau^{ \pm} \rightarrow \mu^{ \pm} \gamma$, Phys. Rev. Lett. 104, 021802 (2010), doi:10.1103/PhysRevLett.104.021802.

[19] B. Aubert et al., Search for lepton flavor violating decays $\tau^{ \pm} \rightarrow \ell^{ \pm} \pi^{0}, \ell^{ \pm} \eta, \ell^{ \pm} \eta^{\prime}$, Phys. Rev. Lett. 98, 061803 (2007), doi:10.1103/PhysRevLett.98.061803.

[20] Y. Miyazaki et al., Search for lepton flavor violating $\tau^{-}$decays into $\ell^{-} \eta, \ell^{-} \eta^{\prime}$ and $\ell^{-} \pi^{0}$, Phys. Lett. B 648, 341 (2007), doi:10.1016/j.physletb.2007.03.027.

[21] B. Aubert et al., Search for lepton flavor violating decays $\tau \rightarrow l^{-} K_{s}^{0}$ with the BABAR experiment, Phys. Rev. D 79, 012004 (2009), doi:10.1103/PhysRevD.79.012004.

[22] Y. Miyazaki et al., Search for lepton flavor violating $\tau^{-}$decays into $\ell^{-} K_{s}^{0}$ and $\ell^{-} K_{s}^{0} K_{s}^{0}$, Phys. Lett. B 692, 4 (2010), doi:10.1016/j.physletb.2010.07.012.

[23] Y. Miyazaki et al., Search for lepton-flavor and lepton-number-violating $\tau \rightarrow \ell h h^{\prime}$ decay modes, Phys. Lett. B 719, 346 (2013), doi:10.1016/j.physletb.2013.01.032.

[24] S. Weinberg, Baryon- and lepton-nonconserving processes, Phys. Rev. Lett. 43, 1566 (1979), doi:10.1103/PhysRevLett.43.1566.

[25] B. Grzadkowski, M. Iskrzyński, M. Misiak and J. Rosiek, Dimension-six terms in the Standard Model Lagrangian, J. High Energ. Phys. 10, 085 (2010), doi:10.1007/JHEP10(2010)085.

[26] G. M. Pruna and A. Signer, The $\mu \rightarrow$ er decay in a systematic effective field theory approach with dimension 6 operators, J. High Energ. Phys. 10, 014 (2014), doi:10.1007/JHEP10(2014)014.

[27] G. M. Pruna, Leptonic CP violation in the charged sector and Effective Field Theory approach, Proc. Sci. 304, 16 (2017), doi:10.22323/1.304.0016.

[28] E. E. Jenkins, A. V. Manohar and M. Trott, Renormalization group evolution of the Standard Model dimension six operators II: Yukawa dependence, J. High Energ. Phys. 01, 035 (2014), doi:10.1007/JHEP01(2014)035.

[29] R. Alonso, E. E. Jenkins, A. V. Manohar and M. Trott, Renormalization group evolution of the Standard Model dimension six operators III: gauge coupling dependence and phenomenology, J. High Energ. Phys. 04, 159 (2014), doi:10.1007/JHEP04(2014)159.

[30] E. E. Jenkins, A. V. Manohar and P. Stoffer, Low-energy effective field theory below the electroweak scale: operators and matching, J. High Energ. Phys. 03, 016 (2018), doi:10.1007/JHEP03(2018)016.

[31] A. Crivellin, S. Davidson, G. M. Pruna and A. Signer, Renormalisation-group improved analysis of $\mu \rightarrow e$ processes in a systematic effective-field-theory approach, J. High Energ. Phys. 05, 117 (2017), doi:10.1007/JHEP05(2017)117.

[32] W. Dekens, E. E. Jenkins, A. V. Manohar and P. Stoffer, Non-perturbative effects in $\mu \rightarrow e \gamma$, J. High Energ. Phys. 01, 088 (2019), doi:10.1007/JHEP01(2019)088.

[33] A. Crivellin, M. Ghezzi, L. Panizzi, G. Marco Pruna and A. Signer, Low- and high-energy phenomenology of a doubly charged scalar (2018), arXiv:1807.10224.

[34] L. Calibbi and G. Signorelli, Charged lepton flavour violation: An experimental and theoretical introduction, Riv. Nuovo Cim. 02, 71 (2018), doi:10.1393/ncr/i2018-10144-0. 
[35] A. Signer, Charged lepton flavor violation, Proc. Sci. 330, 4 (2018), doi:10.22323/1.330.0004. 